\title{
Safety Measurements for Heating of Instruments for Cardiovascular Interventions in Magnetic Particle Imaging (MPI) - First Experiences
}

\author{
Robert L. Duschka ${ }^{1 *}$, Hanne Wojtczyk ${ }^{2}$, Nikolaos Panagiotopoulos ${ }^{1}$, Julian \\ Haegele$^{1}$, Gael Bringout ${ }^{2}$, Thorsten M. Buzug ${ }^{2}$, Joerg Barkhausen ${ }^{1}$ \\ and Florian M. $\operatorname{Vogt}^{\mathbf{1}}$ \\ ${ }^{1}$ Department of Radiology and Nuclear Medicine, University Hospital Schleswig- \\ Holstein, Luebeck, Germany. \\ ${ }^{2}$ Institute of Medical Engineering, University of Luebeck, Luebeck, Germany.
}

Submitted May 2013. Accepted for publication October 2013.

\begin{abstract}
Magnetic particle imaging (MPI) has emerged as a new imaging method with the potential of delivering images of high spatial and temporal resolutions and free of ionizing radiation. Recent studies demonstrated the feasibility of differentiation between signal-generating and non-signalgenerating devices in Magnetic Particle Spectroscopy (MPS) and visualization of commercially available catheters and guide-wires in MPI itself. Thus, MPI seems to be a promising imaging tool for cardiovascular interventions. Several commercially available catheters and guide-wires were tested in this study regarding heating. Heating behavior was correlated to the spectra generated by the devices and measured by the MPI. The results indicate that each instrument should be tested separately due to the wide spectrum of measured temperature changes of signalgenerating instruments, which is up to $85^{\circ} \mathrm{C}$ in contrast to non-signal-generating devices. Development of higher temperatures seems to be a limitation for the use of these devices in cardiovascular interventions.
\end{abstract}

Keywords: magnetic particle imaging (mpi), cardiovascular intervention, safety aspects and heating, interventional devices, magnetic field

\section{INTRODUCTION}

Cardiovascular interventions have emerged as the minimally invasive therapy of choice for many diseases in clinical routine, e.g., coronary artery disease and myocardial infarction [1]. Today, X-ray fluoroscopy and X-ray-based digital subtraction angiography are predominately used for imaging in cardiovascular interventions. Due to increased indications, the number of such interventions

*Corresponding author: Robert L. Duschka, Department of Radiology and Nuclear Medicine, University Hospital Schleswig-Holstein, Campus Luebeck, Ratzeburger Allee 160, 23538 Luebeck, GERMANY. Tel: +49-451-500-6488, Fax: +49-451-500-6497, E-mail: robert.duschka@uksh.de

Other authors: wojtczyk@imt.uni-luebeck.de; nikolaospanagiotopoulos@googlemail.com; Julian. haegele@uksh.de; bringout@imt.uni-luebeck.de; buzug@imt.uni-luebeck.de; joerg.barkhausen @uksh.de; florian.vogt@uksh.de. 
80 Safety Measurements for Heating of Instruments for Cardiovascular Interventions in Magnetic Particle Imaging (MPI) - First Experiences

performed has been increasing constantly. This fact and the need for repeated cardiovascular interventions in patients, e.g., those with chronic coronary artery disease, lead to an increased exposure to ionizing radiation of patients as well as physicians. Furthermore, X-ray fluoroscopy relies on iodine-based contrast agents and holds the potential risk of complications such as allergic reaction or acute renal failure. Despite these drawbacks, X-ray-fluoroscopy is the gold-standard imaging technique for cardiovascular interventions although other imaging modalities have been evaluated as alternatives.

Cone beam computed tomography (CT) allows three-dimensional imaging [2], but the need for iodized contrast media remains, and the radiation of cone beam CT is higher and its temporal resolution is lower compared to X-ray fluoroscopy. To achieve three-dimensional imaging without exposing patients to ionizing radiation, MRI-guided intravascular interventions could be an alternative to those imaging techniques [3]. However, MRI is not used for intravascular interventions in clinical routine yet, although it seems to be promising [4]. Artifacts of metalliferous clinical instruments are one of the main limitations for using MRI in cardiovascular interventions. Other limitations include the risks of radiofrequency [5-8] and gradient-induced heating [9]. A number of studies showed heating behavior of different commercially available interventional instruments in MRI guided interventions [5, 10-13]. These studies indicated the necessity of safety measurements for guide-wires and catheters to predict possible complications for instruments in intravascular interventions involving electromagnetic-field-based imaging methods.

Magnetic particle imaging (MPI) is a new 3D imaging method, introduced by Gleich and Weizenecker in 2005 [14]. MPI distinguishes itself by being completely free of ionizing radiation in contrast to X-ray fluoroscopy, digital subtraction angiography and $\mathrm{CT}$. The principle is based on the nonlinear magnetic response of superparamagnetic iron oxide nanoparticles (SPIOs) in a sinusoidal oscillating magnetic excitation field. The modality is able to provide high temporal and good spatial resolutions and to directly quantify the spatial distribution of SPIOs [14-18]. This makes MPI a promising alternative to CT-fluoroscopy for cardiovascular interventions [19-21]. One precondition for cardiovascular imaging is in vivo real time imaging [17], i.e., visualization of vasculature and instruments with high temporal and spatial resolutions. Recent studies showed that visualization of devices in MPI is possible by an extinction of signal caused by interventional device itself, or alternatively, by labeling devices with SPIOs for MPI $[22,23]$. Besides being able to visualize the instruments in realtime and with high spatial resolution, knowledge about the characteristics of the instruments with respect to safety in MPI is necessary. The aim of this work is to study the heating behavior of commercially available interventional instruments in MPI.

\section{MATERIALS AND METHODS}

\subsection{MPI Scanner}

MPI is based on the detection of the nonlinear response of iron oxide nanoparticles in an oscillating magnetic excitation field [14]. Using two rings of permanent magnets in a mirror-symmetric set-up (Figure 1b), a static magnetic gradient field, 
the so-called selection field $\left(\mathrm{dB}_{\mathrm{z}} / \mathrm{dz}=2.5 \mathrm{~T} / \mathrm{m}\right.$ and $\left.\mathrm{dB}_{\mathrm{x}} / \mathrm{dx}=\mathrm{dB}_{\mathrm{y}} / \mathrm{dy}=1.25 \mathrm{~T} / \mathrm{m}\right)$, is generated. The selection field exhibits a symmetric point with a zero magnetic field, named the field free point (FFP). At the FFP, the particles' magnetization is free to follow an excitation field. Outside the FFP, however, the particles are saturated and thus unable to follow the excitation field. As a consequence, they do not produce any MPI signal.

To determine the spatial distribution of the magnetic tracer material, MPI measures the magnetization change in the tracer material in a time-varying external magnetic field, the so-called drive field. The drive field itself has a defined amplitude and frequency $\left(\omega_{0}\right)$ and causes a changing magnetization $\mathrm{M}(\mathrm{t})$ of SPIOs which can be measured as an induced signal $\mathrm{s}(\mathrm{t})$ in receiving coils.

The relation between the external magnetic field and the magnetization is non-linear. The induced signal, therefore, includes the excitation frequency $\left(\omega_{0}\right)$ and also harmonics $\left(n \omega_{0}\right)$. The presence of these harmonics indicates the presence of magnetic material and also carries the information about the spatial distribution and the quantity of the magnetic material. Furthermore, for reasonable concentrations, the measured signal is proportional to the SPIO-concentration. To acquire complete volumes, it is necessary to move the FFP through the volume and measure and analyze the harmonics.

(a)

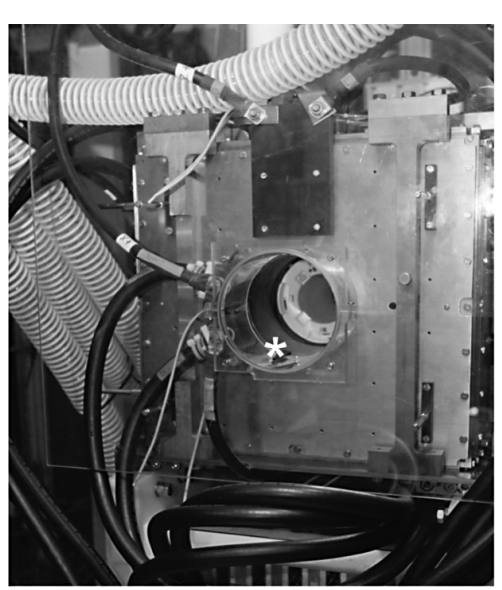

(b)

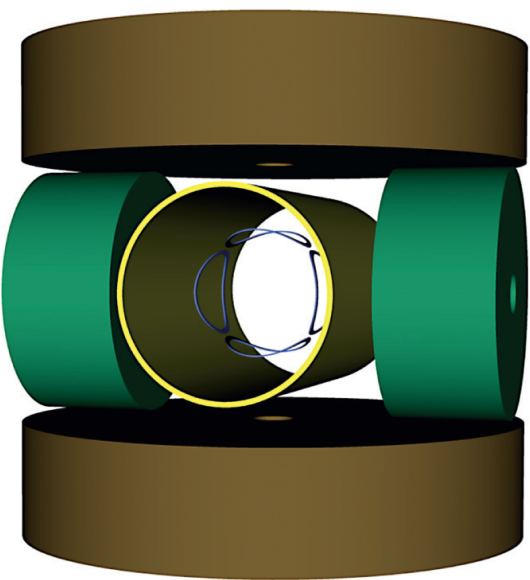

Figure 1. (a) A view of the bore (indicated by *) of the experimental preclinical Magnetic Particle Imaging (MPI) demonstrator at Philips Research Laboratories, Hamburg, Germany. (b) An MPI scanner. The field of view is in the middle of a cylindrical bore (yellow). The brown coils (at the top and bottom) build a selection field and a drive field in vertical direction. Second drive field is generated by the green coils (left and right) in horizontal direction. Third pair of coils which generate a drive field along the bore in the horizontal direction is not shown. Two pairs of receiving coils (purple) are shown inside the bore. 
82 Safety Measurements for Heating of Instruments for Cardiovascular Interventions in Magnetic Particle Imaging (MPI) - First Experiences

This movement of the FFP is realized by drive fields. For this study, three drive field coils are placed in $\mathrm{x}, \mathrm{y}$, and $\mathrm{z}$ directions, respectively, generating oscillating, sinusoidal fields with peak amplitudes $B_{D x}=B_{D y}=B_{D z}=11 \mathrm{mT}$ at frequencies of 25.3, 26.0 and $24.5 \mathrm{kHz}$ for the $\mathrm{x}, \mathrm{y}$ and $\mathrm{z}$ channels, respectively. By moving the FFP through the complete field of view in three dimensions, it would be also possible to acquire threedimensional images.

A more detailed description into MPI is given by Gleich and Weizenecker [14-17]. The setup used for this study, a preclinical MPI demonstrator, is presented in more details in Figure 1 and [24].

\subsection{Interventional Devices}

Based on studies of Haegele et al. [22, 23], eight commercially available interventional instruments were selected: 5 guide-wires and 3 catheters. Three of the 5 guide-wires were made of stainless steel (Amplatz Super Stiff 0.035", Cook Tapered Tefcor 0.035" and Boston Scientific, Platinum Plus, 0.018"), and the other two were made of Nitinol (Terumo Radifocus 0.035 " and Terumo Progreat 0.021 "). Two of the three catheters had a non-braided Tungsten-tip but a braided shaft, which was made of stainless steel (Boston Imager II 5F and Cordis Tempo Aqua 4F). The third catheter, Terumo Radifocus Glidecath 5F (Simmons/Sidewinder), was composed of double-braided stainless steel with hydrophilic coating. Interventional devices differed in composed materials, radiopaque markers and signal response in Magnetic Particle Spectroscopy (MPS), as shown previously [22]. A more detailed description of used interventional devices and their characteristics in MPI is listed in Table 1.

\subsection{Positioning of the Instruments and Temperature Monitoring}

A phantom of 30-cm length, made of acrylic glass and shaped to precisely fit into the bore of the MPI demonstrator, was used to ensure reproducible positioning of the devices in the bore of the setup. The phantom did not show any paramagnetic characteristics and thus did not contribute to the MPI signal. All instruments were inserted into the phantom through individual rubber-made introducers at the top of the phantom and each was positioned at the desired location by a custom-made positioning system (Figure 2). The catheters and guide-wires were inserted and positioned at the center of the phantom, while the temperature sensors were inserted peripherally and placed alongside the instrument at the desired points of temperature measurement. Three temperature sensors were placed at the tip of the interventional devices and at 5 and $10 \mathrm{~cm}$ distance from the tip. The fourth temperature sensor was placed inside the phantom without any contact to other sensors or interventional devices, to serve as a reference (Figure 2) for the temperature in the bore of the MPI demonstrator. The phantom was placed in the middle of the bore. Interventional devices were aligned in the longitudinal direction of the bore. Sinusoidal oscillating magnetic field was switched on continuously during placement of phantom and subsequent measurements. 
Table 1. Different interventional devices in MPI, their radiopaque marker and material, and their potential to generate spectra in MPI in contrast to their heating behavior

\begin{tabular}{|c|c|c|c|c|c|}
\hline & Name & Device/Size & $\begin{array}{c}\text { Radiopaque } \\
\text { marker/ } \\
\text { Material }\end{array}$ & $\begin{array}{c}\text { Signal } \\
\text { response } \\
\text { in MPI }\end{array}$ & $\begin{array}{c}\mathbf{T}_{\max } \\
\text { in MPI }\end{array}$ \\
\hline \multicolumn{6}{|c|}{ Guide-Wires } \\
\hline & $\begin{array}{l}\text { Boston Scientific, } \\
\text { Amplatz Super } \\
\text { Stiff TM }\end{array}$ & $\begin{array}{l}\text { Guide-Wire } \\
0.035 "\end{array}$ & $\begin{array}{l}\text { Stainless steel, } \\
\text { PTFE coating }\end{array}$ & Yes & $84.2^{\circ} \mathrm{C}$ \\
\hline & $\begin{array}{l}\text { Boston Scientific, } \\
\text { Platinum Plus TM }\end{array}$ & $\begin{array}{l}\text { Guide-Wire } \\
0.018 "\end{array}$ & Stainless steel & Yes & $22.0^{\circ} \mathrm{C}$ \\
\hline & $\begin{array}{l}\text { Cook Medical } \\
\text { Tapered Tefcor }\end{array}$ & $\begin{array}{l}\text { Guide-Wire } \\
0.035 "\end{array}$ & $\begin{array}{l}\text { TFE coated } \\
\text { stainless steel }\end{array}$ & Yes & $53.1^{\circ} \mathrm{C}$ \\
\hline & $\begin{array}{l}\text { Terumo Radifocus }{ }^{\circledR} \\
\text { M Standard Type }\end{array}$ & $\begin{array}{l}\text { Guide-Wire } \\
0.035 "\end{array}$ & $\begin{array}{l}\text { Nitinol, } \\
\text { polyurethane, } \\
\text { Tungsten }\end{array}$ & None & $22.0^{\circ} \mathrm{C}$ \\
\hline & $\begin{array}{l}\text { Terumo } \\
\text { Progreat }^{\circledR}\end{array}$ & $\begin{array}{l}\text { Guide-Wire } \\
0.021 "\end{array}$ & $\begin{array}{l}\text { Nitinol, } \\
\text { gold coil }\end{array}$ & None & $22.0^{\circ} \mathrm{C}$ \\
\hline \multicolumn{6}{|l|}{ Catheters } \\
\hline & $\begin{array}{l}\text { Boston Imager }{ }^{\mathrm{TM}} \\
\text { II }\end{array}$ & $\begin{array}{l}\text { Catheter 5F } \\
\text { (Vertebralis) }\end{array}$ & $\begin{array}{l}\text { Tip: non- } \\
\text { braided; Shaft: } \\
\text { stainless steel } \\
\text { Radiopaque } \\
\text { marker: Tungsten }\end{array}$ & Yes & $22.0^{\circ} \mathrm{C}$ \\
\hline & $\begin{array}{l}\text { Cordis TEMPO } \\
\text { AQUA }^{\circledR}\end{array}$ & $\begin{array}{l}\text { Catheter 5F } \\
\text { (Pigtail) }\end{array}$ & $\begin{array}{l}\text { Tip: non- } \\
\text { braided; Shaft: } \\
\text { stainless steel } \\
\text { Radiopaque } \\
\text { marker: Tungsten }\end{array}$ & Yes & $34.2^{\circ} \mathrm{C}$ \\
\hline & $\begin{array}{l}\text { Terumo Radifocus } \\
\text { Glidecath }^{\circledR}\end{array}$ & $\begin{array}{l}\text { Catheter 5F } \\
\text { (Simmons) }\end{array}$ & Polyurethane & None & $22.0^{\circ} \mathrm{C}$ \\
\hline & & & $\begin{array}{l}\text { Double braided } \\
\text { stainless steel }\end{array}$ & & \\
\hline
\end{tabular}

Temperature monitoring was performed continuously using a four-channel Gallium-Arsenide (GaAs)-based fiber optic temperature measuring system (FOTEMP4, Optocon AG, Dresden, Germany). Temperatures were shown on an LCD display and transmitted via RS-232 communication port for real-time data acquisition 


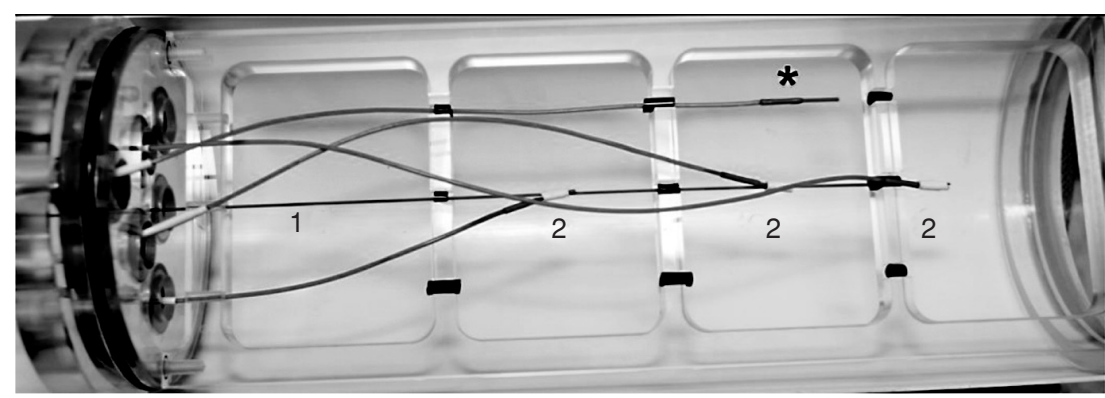

Figure 2. Phantom made of acrylic glass with denoted positions of fiber-optic temperature sensors (2) along the guide-wire (1) at the center of the phantom. The $(*)$ denotes an additional sensor used as a reference without any contact to other sensors or guide-wires.

using FOTEMP-Assistant software for the Win7 operating system of a laptop PC. During excitation, temperature was monitored and recorded every 0.5 seconds for every position for each tested device. Data acquisition was performed until the temperatures reached steady state for more than 2 minutes. Additionally, thermography monitoring was performed by using a thermographic camera (testo $875-2 \mathrm{i}$ set, Testo AG, Lenzkirch, Germany).

\section{RESULTS}

Outside the scanner, the room temperature was at $26^{\circ} \mathrm{C}$. The temperature inside the MPI demonstrator was measured at a constant $22^{\circ} \mathrm{C}$, maintained by an integrated cooling system. Consequently, the reference sensor was also cooled down to $22^{\circ} \mathrm{C}$ in all cases, starting from the moment when the phantom was placed inside the scanner. Only one of the three other sensors attached to the investigated device along the longitudinal axis of the bore was at the field free point (FFP) at one time. The other two sensors, which were 5 and $10 \mathrm{~cm}$ distant from the area of the FFP, cooled down to $22^{\circ} \mathrm{C}$ in all cases, comparable to the reference sensor. Temperature measurements at the sensors attached to the part of the instrument, which was placed at the FFP, showed significantly different, device-specific behavior than the sensors away from the FFP.

\subsection{Non-signal-generating devices}

All non-signal-generating devices showed similar heating characteristics during MPI operation. Both Terumo Guide-wires and Terumo Radifocus Glidecath catheter did not show any temperature increase. Each of the three attached sensors cooled down to $22^{\circ} \mathrm{C}$, compared to the measured $26^{\circ} \mathrm{C}$ room temperature outside the scanner (Figure 3a). No increase of temperature was observed, particularly not at the FFP for any device during the entire measurement. The duration of the cooling process was less than 4 minutes for all three devices. It was not possible to detect any hot spot along the guide-wires and 
(a)

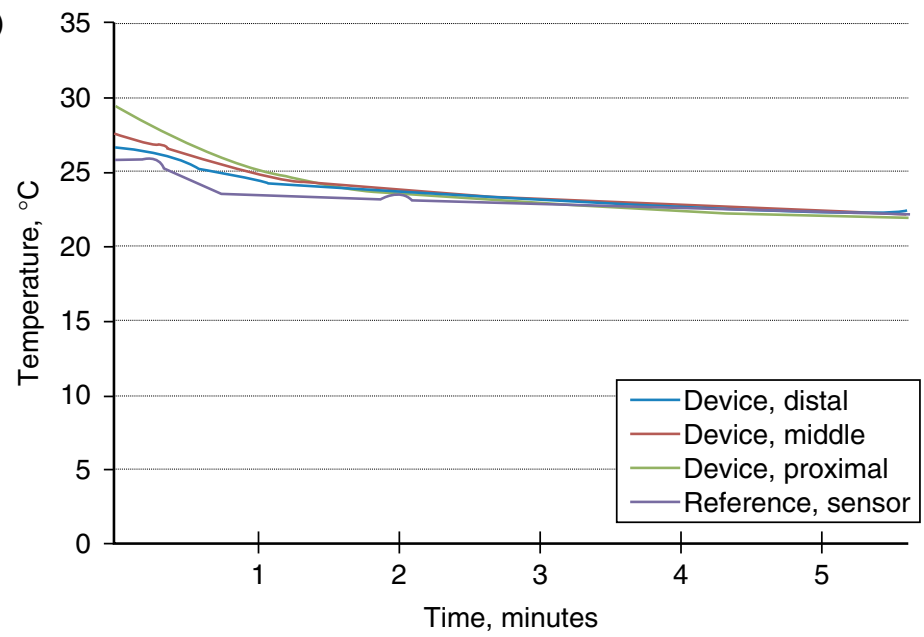

(b)

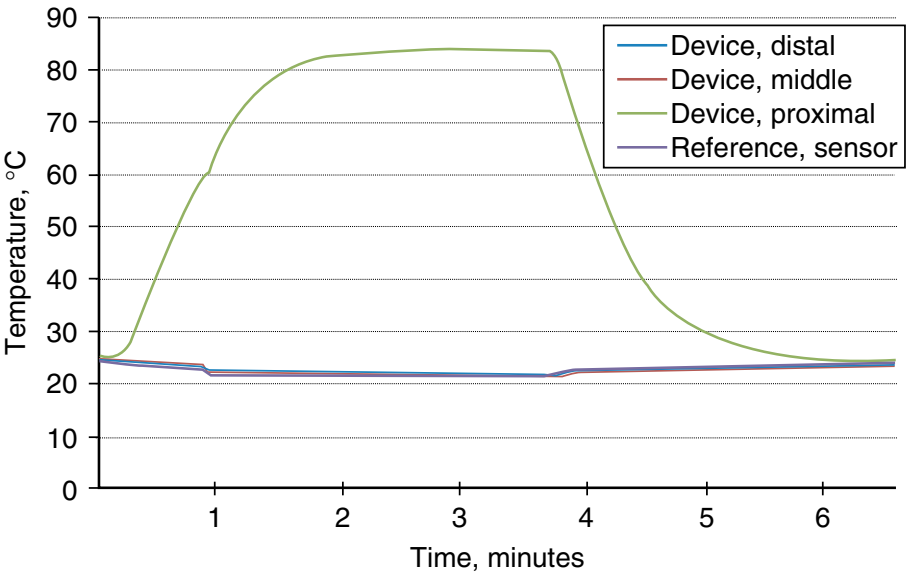

Figure 3. (a) Temperature change of a non-signal-generating device. The observed cooling is due to the low temperature inside the imaging setup, generated by a dedicated cooling system. (b) Temperature change of a signal-generating device with a peak of $85^{\circ} \mathrm{C}$ reached within 2 minutes.

catheters using the thermographic camera (Figure 4). Simultaneously measured MPI signals were similar to the reference MPI signal of the empty setup (Figure $5 \mathrm{a}, \mathrm{b}$ ), again confirming the non-signal-generating characteristics of the tested devices [22].

\subsection{Signal-generating devices}

Although, generally, signal generating devices showed a detectable MPI signal (Figure $5 \mathrm{c}$ ), different heating patterns were recorded. Differences were observed in the magnitude of peak temperatures and peak times. 
86 Safety Measurements for Heating of Instruments for Cardiovascular Interventions in Magnetic Particle Imaging (MPI) - First Experiences

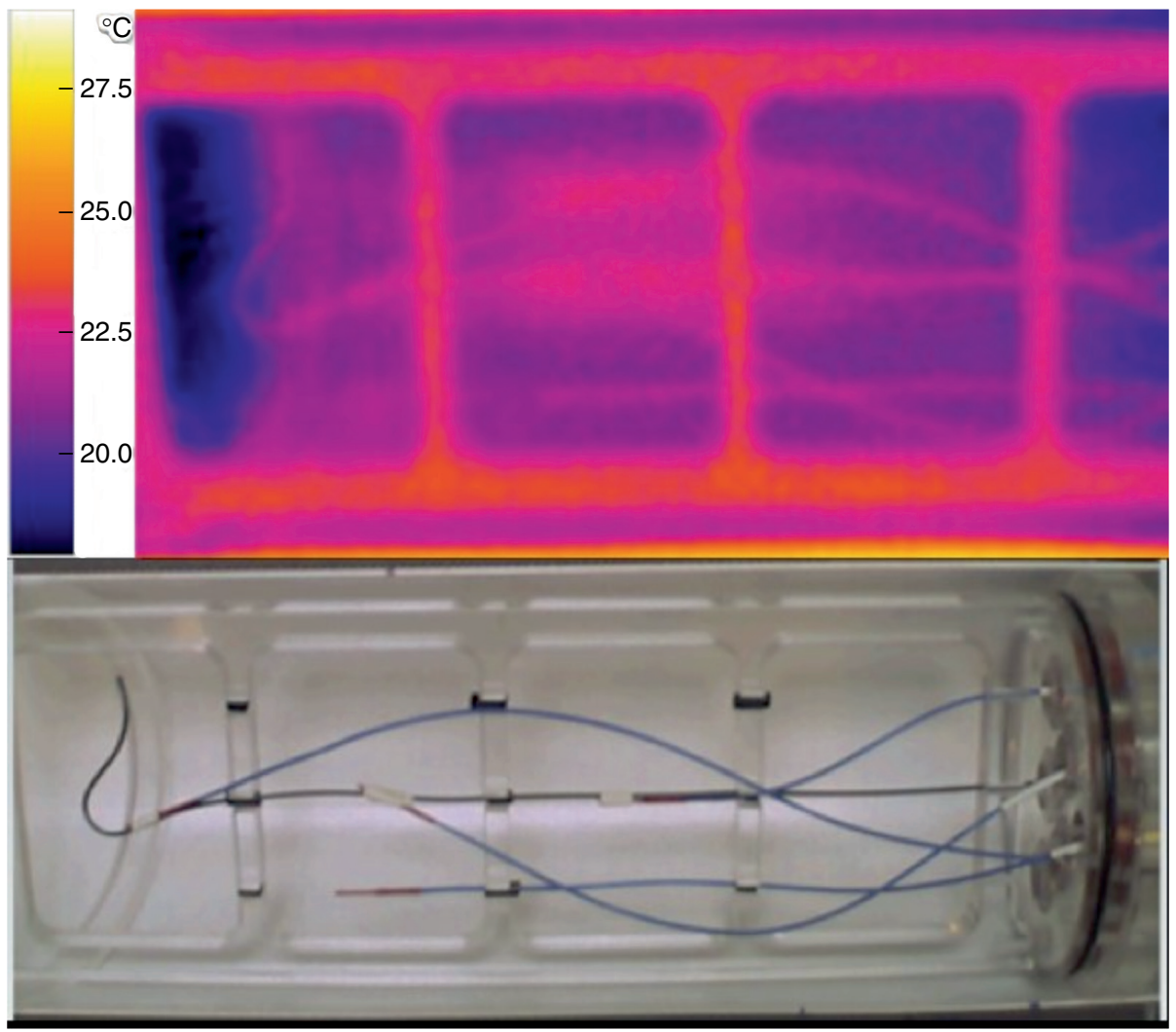

Figure 4. Thermograph of a non-signal-generating device (above, right) with temperatures of approximately $22^{\circ} \mathrm{C}$ (above, left). The position of the fiber-optic sensor corresponds to the field free point (FFP) inside the scanner. No hot spot or heating can be observed.

For all devices showing heating inside the demonstrator, heating at the FFP started at the moment the device was placed in the bore of the scanner. The highest temperature was observed for the Amplatz Super Stiff Guide-Wire, which reached $85^{\circ} \mathrm{C}$ within 2 minutes at the FFP (Figure $3 b$ ). The cool-down time for the guidewire when it was removed from sinusoidal oscillating magnetic field was nearly as short as the time that was needed to heat up. Fifty-five seconds after removing the Amplatz Superstiff from the FFP, the temperature was lowered to $37^{\circ} \mathrm{C}$. One minute later, the sensor detected $26^{\circ} \mathrm{C}$ at the former hot spot. The longest time to peak was observed for the shaft of Boston Imager II Pigtail catheter ( $3 \mathrm{~min}$ ). The maximum temperature was $34.2^{\circ} \mathrm{C}$. The tip did not show any heating at all when it was placed at the FFP. Cook Tapered Tefcor guide-wire 0.035" achieved a maximum temperature of $52.3^{\circ} \mathrm{C}$. The data from the thermographic camera proved that all 

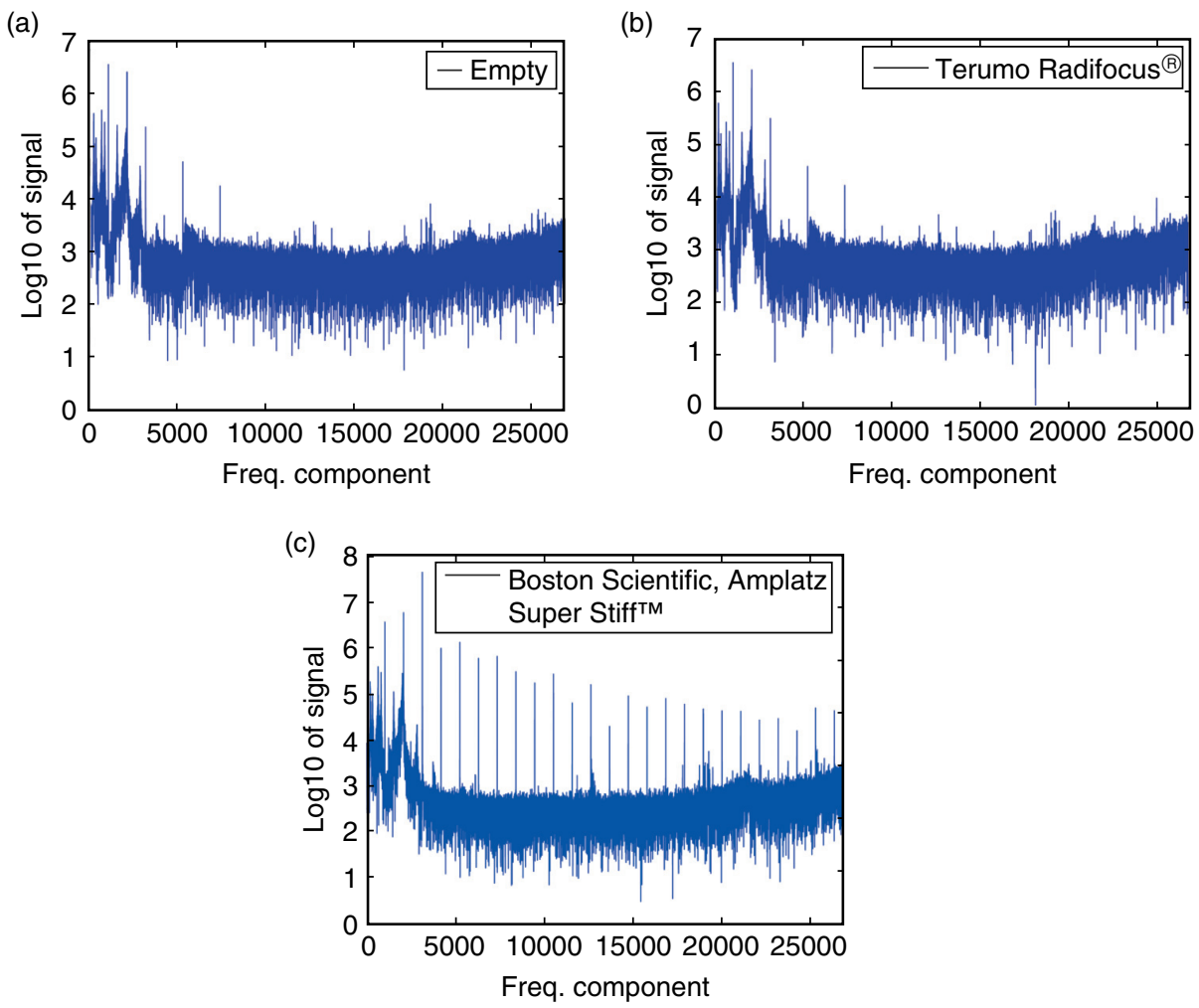

Figure 5. (a) Measured signal with no material inside the bore of the imaging setup, used as a reference background for the assessment of signalgenerating and non-signal generating devices. (b) Measured signal of Terumo Radifocus ${ }^{\circledR}$ M Standard Type, a non-signal-generating device, similar to the reference without peaks. (c) Measured signal of Boston Scientific Amplatz Super Stiff TM, a signal-generating device, showing amplitude peaks.

devices featured punctiform hot spots exclusively at the FFP and no heating at all away from the FFP (Figure 6). When the temperature sensors were removed from the FFP, temperatures decreased to $22^{\circ} \mathrm{C}$, the ambient temperature inside the MPI demonstrator.

Two of the tested signal-generating devices, the Boston Scientific Platinum PLUS guide-wire and the Boston Imager ${ }^{\mathrm{TM}}$ II catheter, showed no increase in temperature, neither at the FFP nor in its vicinity, although they showed a detectable MPI signal. Thus, the thermal behavior of these two instruments did not correlate with the former categorization of signal-generating and non-signal-generating instruments [22]. 
88 Safety Measurements for Heating of Instruments for Cardiovascular Interventions in Magnetic Particle Imaging (MPI) - First Experiences

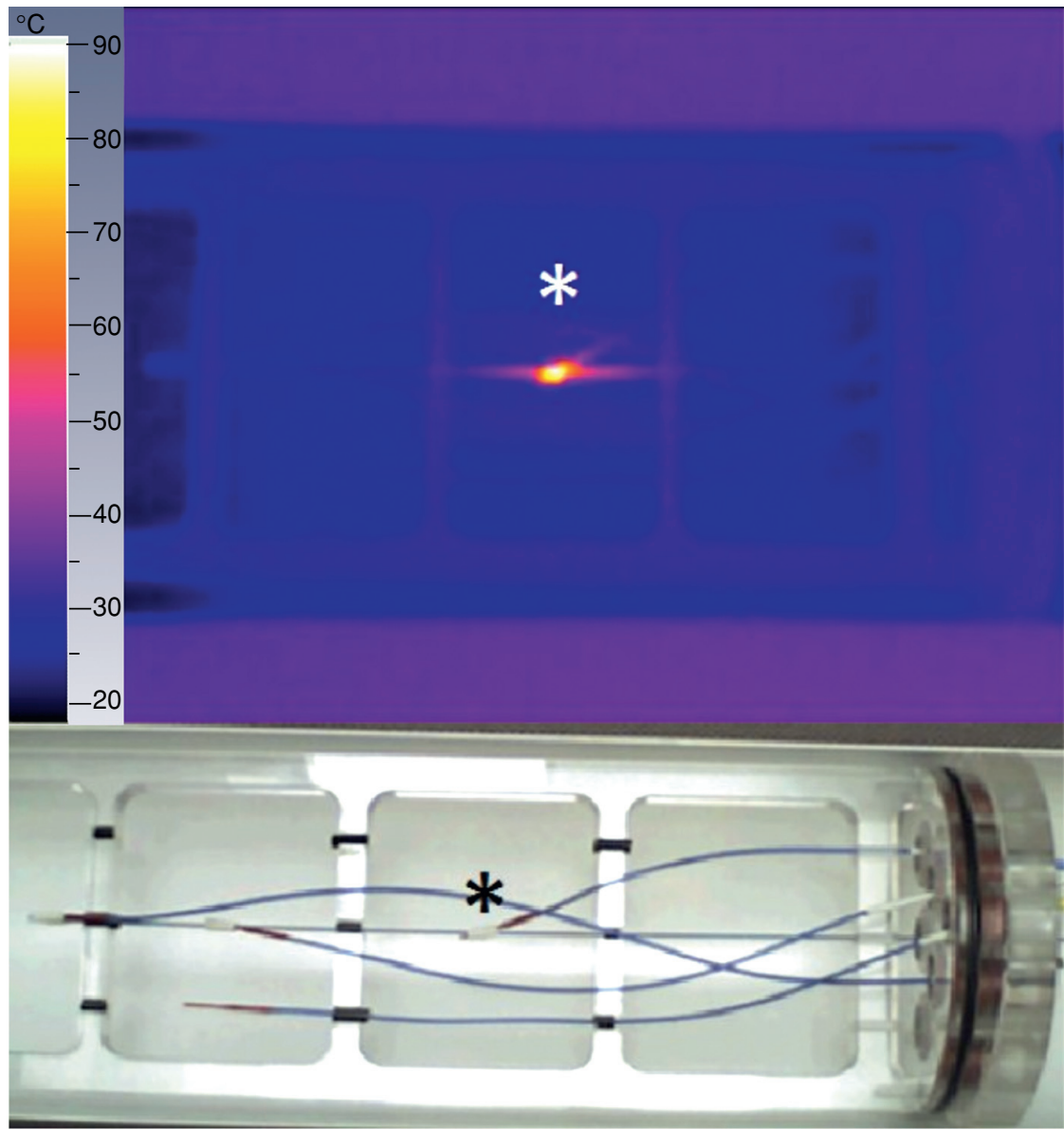

Figure 6. Thermograph of a signal-generating device (above). The fiber-optic temperature sensor is located at the hot spot $(*)$, which is at the field free point (FFP) inside the setup. Other sensors did not measure any heating and no other hot spots were detected along the guide-wire.

\section{DISCUSSION}

This study provides, to the best of our knowledge, the first security measurement regarding heating of interventional instruments in MPI. The selection of evaluated instruments was based on the most utilized instruments in our institution as well as on results from prior studies of Haegele et al. in 2012 [22]. Haegele was able to distinguish between "signal-generating" and "non-signal-generating" devices. Non-signalgenerating instruments did not show any temperature increase at all, either at the FFP or in its vicinity. Terumo guide-wire ("M standard type") does not contain any distal radiopaque tip marker and its core material is Nitinol. Different from the guide-wire " $M$ 
standard type," the Terumo "Progreat" has a 3-cm gold coil as a distal radiopaque marker while its core material is Nitinol as well. Based on the utilized material, neither MPI signal nor an increase of temperature was expected.

Terumo Radifocus "Glidecath" catheter contains a double-braided stainless steel core with a polyurethane jacket. Although the device contains stainless steel, no remarkable signal was generated, as Haegele et al. has already shown. However, there was no temperature rise either. As described previously, it is assumed that the composition of metals is responsible for the signal properties and the non-ferromagnetic characteristics of these devices, which may explain no heating. Furthermore, prior to the use of these catheters in an interventional MPI setting, they must be made visible and clearly distinguishable from background noise and from intravascular tracers, which in turn could possibly create new problems.

Several methods of labeling non-signal-generating devices with SPIOs have been described previously and have shown promising results [22, 23]. In general, active and passive visualization approaches can be distinguished. The latter seems to be more easily accessible and can be achieved by oppositional passive opacification of the vessel lumen using intravascular iron-oxide contrast agent [22, 23]. Their contrast mechanism relies on the cancellation of signals by the interventional instruments themselves.

In contrast, the active visualization of non-signal-generating instruments is also a promising approach. One possibility to actively generate a signal is filling of existing voids, i.e., balloons, within the catheter with SPIOs solutions. Labeling of devices with an SPIO-based enamel is also feasible and has been described in a previous study [22]. However, heating behavior of those SPIO coated devices is not yet known. Indeed, an AC magnetic field holds the risk of inducing current, e.g., for conductive devices which have ferromagnetic characteristics. However, the development of hazardously high temperatures caused by SPIO labeled devices seems improbable as the needed concentrations of SPIOs will most likely not be high enough.

Regarding the results of the signal-generating instruments, it seems to be plausible to categorize these instruments into two sub-groups. One sub-group includes those devices which show the expected heating due to integrated ferromagnetic material and those which do not show any heating at all, although they generate a signal in MPI. However, the majority of catheters showed a temperature rise. One catheter showed an increase up to $85^{\circ} \mathrm{C}$ and therefore seems not suitable for cardiovascular interventions due to safety concerns, i.e., intravascular protein denaturation, which can result in thrombogenic events or damage of the vascular wall. Intravascular cooling might mitigate the heating effects of these interventional devices in vivo, but has not been tested so far. Overall, it seems to be necessary to discriminate non-signal-generating instruments and signal-generating instruments without heating from those signalgenerating instruments causing heating.

Ferromagnetic characteristics might seem to be the main reason for generation of an MPI signal in the devices listed above. These characteristics lead to hysteresis losses while changing magnetization in sinusoidal magnetic fields. Most likely, the broad range of heating may result from different hysteresis losses due to different ferromagnetic behavior of compositions of metal used as radiopaque markers, and those 

in Magnetic Particle Imaging (MPI) - First Experiences

different compositions may result in different ferromagnetic characteristics. The fact that some catheters which generate signal in MPI do not show any heating remains unclear. A possible explanation is the presence of eddy currents, which are well known from MRI. Eddy currents are generated in magnetic fields in metalliferous implants. It may be possible that these currents are the reason for the MPI-signal detected in some devices (e.g., Boston Scientific Platinum PLUS guide-wire and Cordis Tempo Aqua Vertebralis catheters) without inducing any heating at all in opposition to the devices with ferromagnetic characteristics which show both MPI signal and heating. Eddy currents can cause detectable MPI-signals without heating. In contrast, eddy currents result in heating if current flow is influenced by high resistance, e.g., in contact points of different materials in devices. Furthermore, it seems possible that the induction of eddy currents could influence heating behavior of metalliferous devices even more for other frequencies than $25 \mathrm{kHz}$, as they were used in this study. For instruments which do not show any MPI signal, the situation still seems to be unclear. Heating of instruments without any observed signal in MPI does not seem to be plausible at first, yet it is not impossible. In theory, heating caused by eddy currents is also possible in non-signal-generating devices, especially when complex devices (e.g., metalliferous stents) are used. In those cases, linear eddy currents could be induced and a measurable MPI signal could be missed. Taking heating due to eddy currents into account, heating of non-signal-generating devices seem to be possible. This suggests the necessity of further studies for non-signal-generating devices at different frequencies.

Recent studies indicate the risk of peripheral nerve stimulation using MPI at frequencies of $25 \mathrm{kHz}$ [25] while a secure threshold seems to be achievable at frequencies above $100 \mathrm{kHz}$ [26]. Due to limitations related to peripheral nerve stimulation thresholds and tissue heating, future MPI scanners may operate at frequencies different from $25 \mathrm{kHz}$. Thus, instruments should be tested for all relevant excitation frequencies, which may range from 5 to $500 \mathrm{kHz}$.

In fact, heating behavior in MPI is a complex phenomenon which is not fully understood to date. The behavior of devices in MPI seems to be dependent on both ferromagnetic characteristics and composition of devices; thus, it is not possible to reliably predict whether an instrument that contains ferromagnetic material will show a rise in temperature in MPI or not.

Concerning safety aspects, several studies showed different behavior of different interventional instruments in MRI and declared the necessity of safety/heating measurements for each available device [5, 10-13]. The results of this study suggest further studies to predict the risk of interventional instruments for cardiovascular applications in MPI. Furthermore, each device should be tested individually before being used in interventional applications in MPI, as this study clearly shows that inconclusive and unpredictable heating characteristics for signal-generating instruments exist.

\section{CONCLUSIONS}

Cardiovascular interventions seem to be a promising application for MPI, providing good spatial and high temporal resolutions without exposure to ionizing radiation. Besides visualization of interventional instruments, safety aspects are one of the preconditions and a topic of ongoing research. Utilization of instruments containing metal, such as catheters, guide-wires or stents seems to be a limitation for some of the 
signal-generating devices due to their heating behavior in MPI. Cardiovascular interventions seem to be safe and very promising by using non-signal-generating catheters coated or loaded with SPIOS, or those signal-generating devices which do not show any heating behavior in MPI. However, it remains unclear to what extent eddy currents will cause rise in temperature. Consequently, additional experiments must be performed to further study safety aspects for MPI guided cardiovascular interventions.

\section{ACKNOWLEDGEMENTS}

The authors gratefully acknowledge the financial support of the German Federal Ministry of Education and Research (BMBF) under grant number 13N11090 and of the European Union and the State Schleswig-Holstein (Programme for the Future Economy) under grant number 122-10-004. Moreover, the authors express their gratitude towards Philips Technologie GmbH Forschungslaboratorien, Hamburg, Germany for supporting safety measurements using the MPI demonstrator.

\section{CONFLICT OF INTEREST}

The authors indicated no potential conflicts of interest.

\section{NOMENCLATURE}

$B \quad$ magnetic (induction) field, T

$B_{\mathrm{Dx}} \quad$ amplitude of magnetic drive field in x-direction, $\mathrm{T}$

$B_{\text {Dy }} \quad$ amplitude of magnetic drive field in y-direction, $\mathrm{T}$

$B_{\mathrm{Dz}} \quad$ amplitude of magnetic drive field in z-direction, $\mathrm{T}$

FFP Field Free Point

$M(t) \quad$ magnetization (as a function of $\mathrm{t}$ )

$s(t) \quad$ induced signal (as a function of $\mathrm{t}$ )

$t \quad$ time

\section{Greek}

$n \omega_{0} \quad$ frequencies of the harmonics of the excitation frequency

$\omega_{0} \quad$ excitation frequency

\section{REFERENCES}

[1] Eagleton MJ: Intraprocedural imaging: flat panel detectors, rotational angiography, FluoroCT, IVUS, or still the portable C-arm? J Vasc Surg 2010, 52(4 Suppl): 50S-59S.

[2] Wallace MJ, Kuo MD, Glaiberman C, Binkert CA, Orth RC, Soulez G: Three-dimensional C-arm cone-beam CT: applications in the interventional suite. J Vasc Interv Radiol 2008, 19(6): 7 99-813.

[3] Bock M, Wacker FK: MR-guided intravascular interventions: techniques and applications. J Magn Reson Imaging 2008, 27(2): 326-338.

[4] Kagadis GC, Katsanos K, Karnabatidis D, Loudos G, Nikiforidis GC, Hendee WR: Emerging technologies for image guidance and device navigation in interventional radiology. Med Phys 2012, 39(9): 5768-5781.

[5] Martin AJ, Baek B, Acevedo-Bolton G, Higashida RT, Comstock J, Saloner DA: MR imaging during endovascular procedures: an evaluation of the potential for catheter heating. Magn Reson Med 2009, 61(1): 45-53. 
92 Safety Measurements for Heating of Instruments for Cardiovascular Interventions in Magnetic Particle Imaging (MPI) - First Experiences

[6] Nitz WR, Oppelt A, Renz W, Manke C, Lenhart M, Link J: On the heating of linear conductive structures as guide wires and catheters in interventional MRI. J Magn Reson Imaging 2001, 13(1): $105-114$.

[7] Settecase F, Hetts SW, Martin AJ, Roberts TP, Bernhardt AF, Evans L, Malba V, Saeed M, Arenson RL, Kucharzyk W, Wilson MW: RF Heating of MRI-Assisted Catheter Steering Coils for Interventional MRI. Acad Radiol 2011, 18(3): 277-285.

[8] Muranaka H, Horiguchi T, Usui S, Ueda Y, Nakamura O, Ikeda F: Dependence of RF heating on SAR and implant position in a 1.5T MR system. Magn Reson Med Sci 2007, 6(4): 199-209.

[9] Graf H, Steidle G, Schick F: Heating of metallic implants and instruments induced by gradient switching in a 1.5-Tesla whole-body unit. J Magn Reson Imaging 2007, 26(5): 1328-1333.

[10] Wacker FK, Reither K, Ebert W, Wendt M, Lewin JS, Wolf KJ: MR image-guided endovascular procedures with the ultrasmall superparamagnetic iron oxide SH U $555 \mathrm{C}$ as an intravascular contrast agent: study in pigs. Radiology 2003, 226(2): 459-464.

[11] Tzifa A, Krombach GA, Krämer N, Krüger S, Schütte A, von Walter M, Schaeffter T, Qureshi S, Krasemann T, Rosenthal E, Schwartz CA, Varma G, Buhl A, Kohlmeier A, Bücker A, Günther RW, Razavi R: Magnetic resonance-guided cardiac interventions using magnetic resonance-compatible devices: a preclinical study and first-in-man congenital interventions. Circ Cardiovasc Interv 2010, 3(6): 585-592.

[12] Tong N, Shmatukha A, Asmah P, Stainsby J: Practical aspects of MR imaging in the presence of conductive guide wires. Phys Med Biol 2010, 55(1): N13-22.

[13] Saeed M, Hetts SW, English J, Wilson M: MR fluoroscopy in vascular and cardiac interventions (review). Int J Cardiovasc Imaging 2012, 28(1): 117-137.

[14] Gleich B, Weizenecker J: Tomographic imaging using the nonlinear response of magnetic particles. Nature 2005, 435(7046): 1214-1217.

[15] Weizenecker J, Borgert J, Gleich B: A simulation study on the resolution and sensitivity of magnetic particle imaging. Phys Med Biol 2007, 52(21): 6363-6374.

[16] Gleich B, Weizenecker J, Borgert J: Experimental results on fast 2D-encoded magnetic particle imaging. Phys Med Biol 2008, 53(6): N81-84.

[17] Weizenecker J, Gleich B, Rahmer J, Dahnke H, Borgert J: Three-dimensional real-time in vivo magnetic particle imaging. Phys Med Biol 2009, 54(5): L1-L10.

[18] Buzug TM, Bringout G, Erbe M, Gräfe K, Graeser M, Grüttner M, Halkola A, Sattel TF, Tenner W, Wojtczyk H, Haegele J, Vogt FM, Barkhausen J, Ludtke-Buzug K: Magnetic Particle Imaging: Introduction to Imaging and Hardware Realization. Z Med Phys 2012, 22(4): 323-334.

[19] Haegele J, Sattel T, Erbe M, Luedtke-Buzug K, Taupitz M, Borgert J, Buzug TM, Barkhausen J, Vogt FM: [Magnetic particle imaging (MPI)]. Rofo 2012, 184(5): 420-426 [Article in German].

[20] Schütz G: The potential of magnetic particle imaging in the competitive environment of cardiac diagnostics. In: Magnetic Particle Imaging. Edited by Buzug T, Borgert J: SPPHY 140: SpringerVerlag Berlin Heidelberg; 2012: pp. 129-134.

[21] Duschka RL, Haegele J, Panagiotopoulos N, Wojtczyk H, Barkhausen J, Vogt FM, Buzug TM, LüdtkeBuzug K: Fundamentals and Potential of Magnetic Particle Imaging. Curr Cardiovasc Imaging Rep 2013, 6(5): 390-398.

[22] Haegele J, Biederer S, Wojtczyk H, Graeser M, Knopp T, Buzug TM, Barkhausen J, Vogt FM: Toward cardiovascular interventions guided by magnetic particle imaging: First instrument characterization. Magn Reson Med 2013 Jun, 69(6): 1761-1767.

[23] Haegele J, Rahmer J, Gleich B, Borgert J, Wojtczyk H, Panagiotopoulos N, Buzug TM, Barkhausen J, Vogt FM: Magnetic particle imaging: visualization of instruments for cardiovascular intervention. Radiology 2012, 265(3):933-938.

[24] Gleich B, Weizenecker J, Timminger H, Bontus C, Schmale I, Rahmer J, Schmidt J, Kanzenbach J, Borgert J: Fast MPI Demonstrator with Enlarged Field of View. In: International Society for Magnetic Resonance in Medicine, 18th Annual Meeting. vol. 18th Annual Meeting. Stockholm; 2010: 3285. 
[25] Saritas E, Goodwill P, Zhang G, Wenxiao Y, Conolly S: Safety Limits for Human-Size Magnetic Particle Imaging Systems. In: Magnetic Particle Imaging. Edited by Buzug TM, Borgert J, vol. SPPHY 140: Springer-Verlag Berlin Heidelberg; 2012: pp. 325-330.

[26] Weinberg IN, Stepanov PY, Fricke ST, Probst R, Urdaneta M, Warnow D, Sanders H, Glidden SC, McMillan A, Starewicz PM, Reilly JP: Increasing the oscillation frequency of strong magnetic fields above $101 \mathrm{kHz}$ significantly raises peripheral nerve excitation thresholds. Med Phys 2012, 39(5): 2578-2583. 



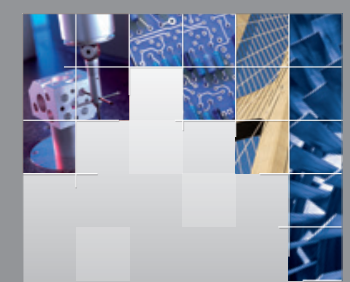

\section{Enfincering}
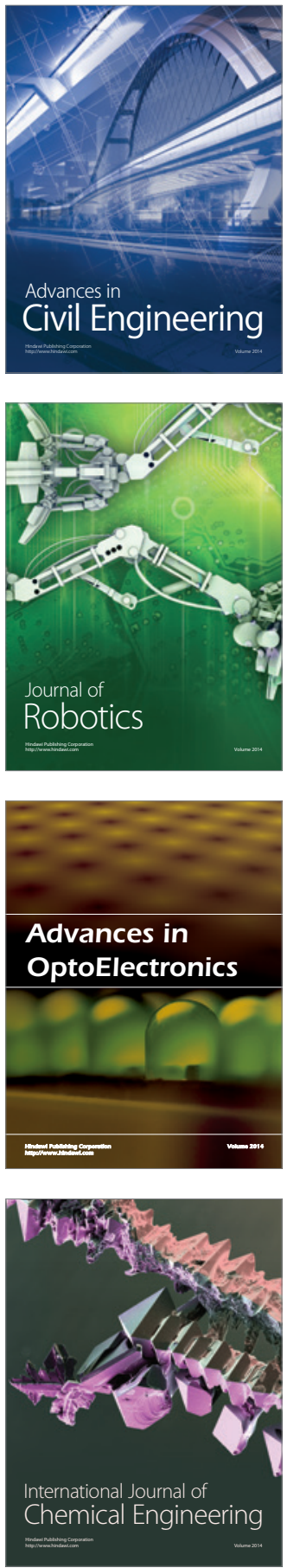

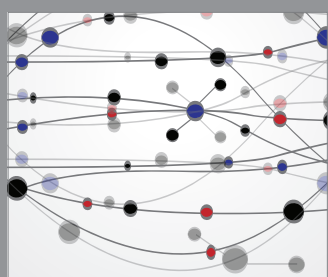

The Scientific World Journal

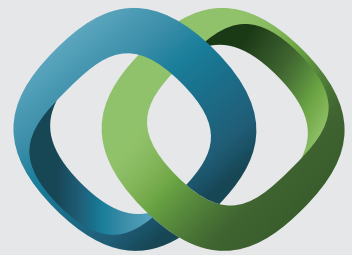

\section{Hindawi}

Submit your manuscripts at

http://www.hindawi.com
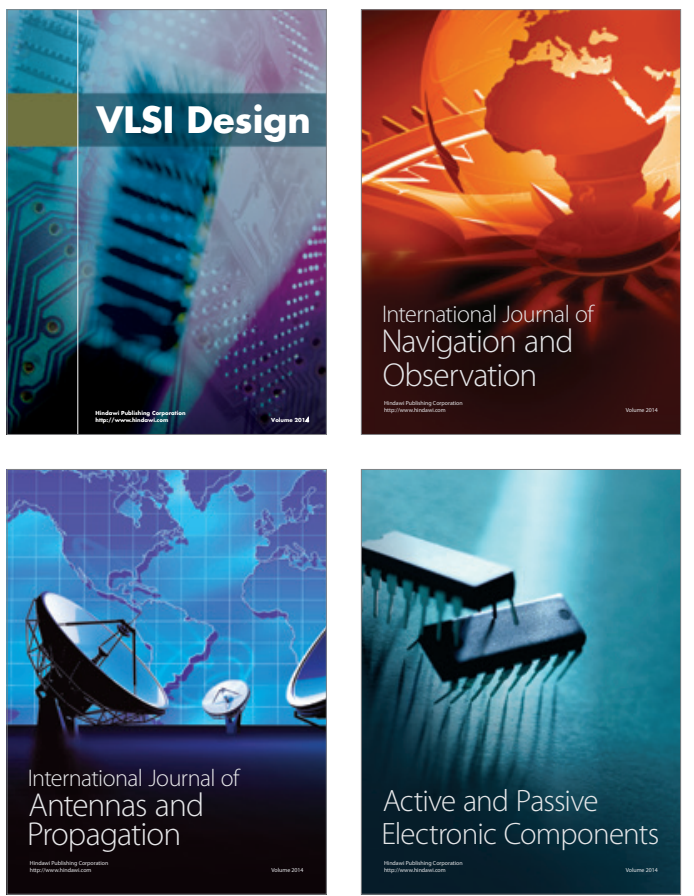
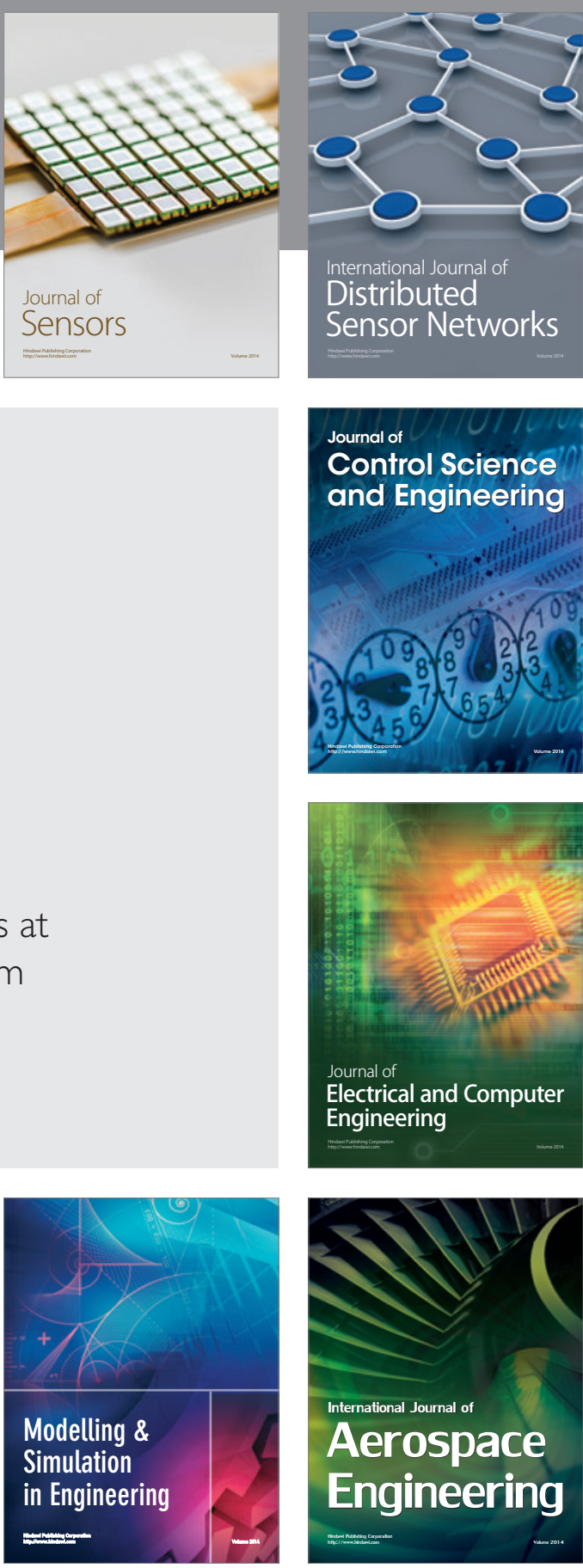

International Journal of

Distributed

Sensor Networks

Journal of

Control Science

and Engineering
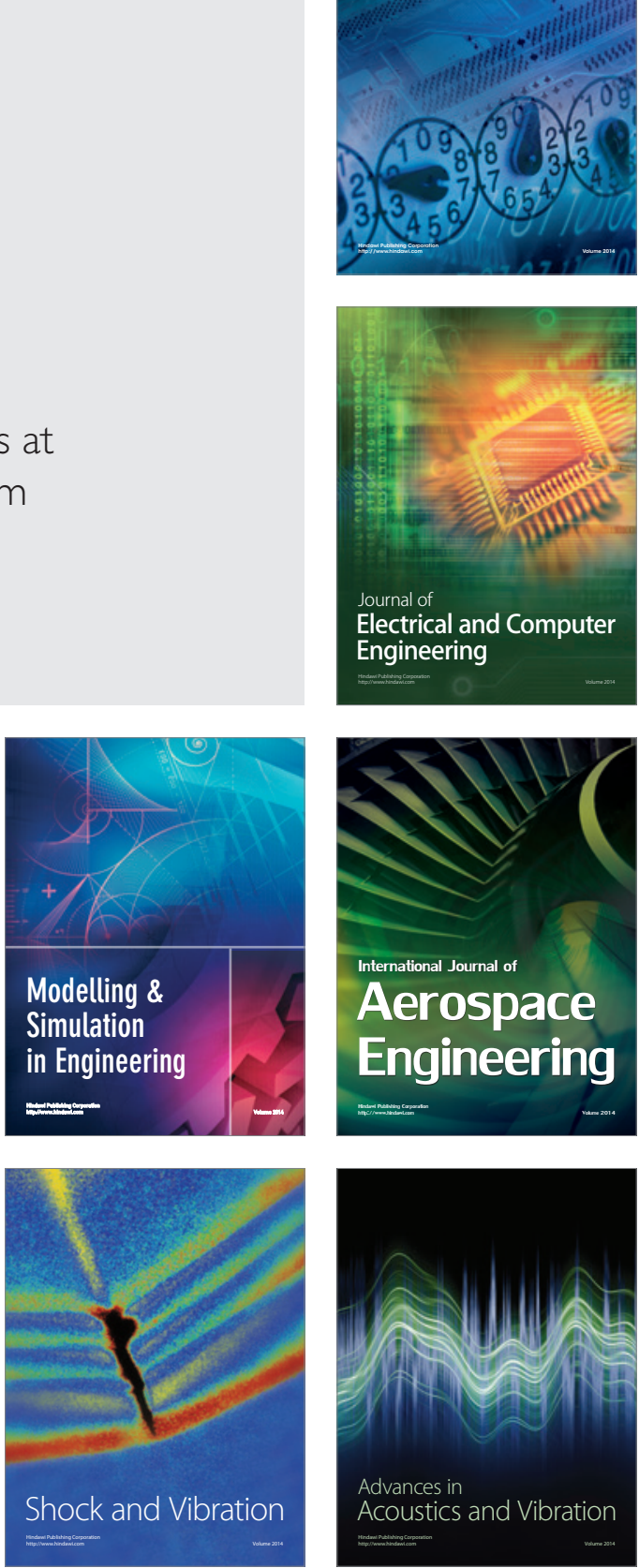